\title{
iência \\ QUALIDADE DO PARTO E IMPACTO NOS \\ INDICADORES DA SAÚDE DA CRIANÇA
}

QUALITY OF BIRTH AND IMPACT ON CHILD HEALTH INDICATORS

Isabelle Christine Fonsêca Gomes de Araújo | | Universidade Federal do Rio Grande do Norte (UFRN) || Enfermeira

E-mail: isabellechristine1@hotmail.com

Tainara Lorena dos Santos Ferreira || Universidade Federal do Rio Grande do Norte (UFRN) || Enfermeira || Mestranda pelo Programa em PósGraduação em Saúde Coletiva da UFRN || E-mail: tainara_lorena@hotmail.com

Daísy Vieira de Araújo || Universidade Federal do Rio Grande do Norte (UFRN) || Enfermeira || Doutoranda pelo Programa de Pós-Graduação em Ciências da Saúde da UFRN | | Professora Adjunto III da FACISA/UFRN | | E-mail: mestredaisy@yahoo.com.br

Káthya Daniella Figueiredo Melo || Enfermeira || E-mail: kathyadaniella@hotmail.com

Fábia Barbosa de Andrade || Universidade Federal do Rio Grande do Norte (UFRN) || Enfermeira || Doutora em Ciências da Saúde || Professora Adjunto IV da UFRN | | E-mail: fabiabarbosabr@gmail.com

Autor responsável pela correspondência

Isabelle Christine Fonsêca Gomes de Araújo || E-mail: isabellechristine1@hotmail.com 


\section{RESUMO}

Introdução: A qualidade da assistência do parto indica um importante fator nas taxas de mortalidade infantil, pois os problemas decorrentes deste, quando realizado de forma inadequada, pode ter repercussão sobre o recém-nascido durante os primeiros dias de vida. Objetivo: Avaliar a qualidade da assistência do parto e a correlação com os indicadores de saúde da criança. Método: Trata-se de um estudo quantitativo e avaliativo dos indicadores de morbidade e mortalidade materno e infantil realizado nas Unidades Básicas de Saúde que integram a rede da Atenção Primária à Saúde (APS), do município de Santa Cruz, Rio Grande do Norte, Brasil. Com uma amostra de 200 mulheres mães de crianças menores de dois anos. Resultados: Foram entrevistadas mães com média de idade de 28,17 anos, com bebês com em média 10,42 meses de idade. 95,5\% das mães afirmaram ter realizado o acompanhamento pré-natal. A maioria (60,5\%) das mulheres classificou a assistência recebida no parto como boa; 74,5 \% afirmaram que a criança foi colocada no peito após o parto; $90 \%$ dos binômios mãe-bebê estiverem juntos no quarto após o parto. Conclusões: Pode-se perceber que a assistência ao parto acontece de forma adequada e satisfatória, atendendo necessidades da maioria das entrevistadas. Porém ainda há uma necessidade de conscientização e qualificação dos profissionais de saúde, para que não ocorram situações indesejáveis na saúde materno-infantil e diminua as taxas de morbidade e mortalidade materna e neonatal.

Palavras-Chave: Parto. Saúde da criança. Serviços de Saúde Materno-Infantil.

\section{ABSTRACT}

Introduction: The quality of delivery care indicates an important factor in the infant mortality rates, since the problems arising from it, when performed in an inappropriate way, can have repercussion on the newborn during the first days of life. Objective: To evaluate the quality of childbirth care and the correlation with child health indicators. Methods: This is a quantitative and evaluative study of the indicators of maternal and infant morbidity and mortality carried out at the Basic Health Units that integrate the Primary Health Care (PHC) network of the municipality of Santa Cruz, Rio Grande do Norte, Brazil. With a sample of 200 women mothers of children under two years. Results: Mothers with a mean age of 28.17 years were interviewed, with infants with a mean of 10.42 months of age. $95.5 \%$ of the mothers reported having had prenatal care. The majority $(60.5 \%)$ of the women classified care received at delivery as good; $74.5 \%$ stated that the child was placed in the breast after delivery; $90 \%$ of the mother-baby binomials are together in the fourth postpartum. Conclusions: It can be noticed that the delivery assistance takes place adequately and satisfactorily, meeting the needs of the majority of the interviewees. However, there is still a need for awareness and qualification of health professionals, so that undesirable situations in maternal and child health do not occur and the rates of maternal and neonatal morbidity and mortality decrease.

s: Parturition. Child Health. Maternal-Child Health Services. 


\section{Introdução}

A qualidade da assistência do parto indica um importante fator nas taxas de mortalidade infantil, pois os problemas decorrentes deste, quando realizado de forma inadequada, pode ter repercussão sobre o recém-nascido durante os primeiros dias de vida, ou seja, quanto melhor a assistência menor será o índice da morbimortalidade materno-infantil.

Dessa forma, é necessária uma atenção humanizada que envolva um conjunto de conhecimentos, práticas e atitudes que visem à promoção do parto, do nascimento saudável e a prevenção da morbimortalidade materna e perinatal. Esta deve ser iniciada no pré-natal, estendendo-se até o puerpério, buscando garantir que os profissionais de saúde realizem procedimentos benéficos para a mulher e o bebê, evitando intervenções desnecessárias e preservando a saúde de ambos.

Quando ocorre mortalidade neonatal precoce, indica causas de óbitos relacionadas principalmente à precária assistência oferecida às mães durante o período do pré-natal e do parto, bem como a atenção inadequada aos recémnascidos nas salas de parto e unidades neonatais ${ }^{1}$.

No Brasil, a Taxa de Mortalidade Neonatal (TMN) caiu de 13,1 em 2000 para 8,1 em 2011 por 1000 nascidos vivos, uma queda 61,8\%. Sobre a Taxa de Mortalidade Infantil (TMI) foi encontrada de 26,1 em 2000 e 15,3 em 2011, o que equivale a uma queda de $58,6 \%$. A mortalidade neonatal também está vinculada a causas preveníveis, relacionadas ao acesso e utilização dos serviços de saúde, além da qualidade da assistência pré-natal, ao parto e ao recém-nascido. As afecções perinatais respondem atualmente por cerca de $60 \%$ das mortes infantis e $80 \%$ das mortes neonatais, além de ser a primeira causa de morte em menores de cinco $\operatorname{anos}^{2}$.

Dessa forma, é importante ressaltar que apesar do declínio dos índices de orbidades e mortalidades infantis no Brasil, ainda assim o impacto da redução 
não está claramente elucidado quanto à eficácia das intervenções sociais e de saúde estabelecidas até a atualidade. Por outro lado, os dados deixam claro que as práticas de atenção à saúde devem ser apoiadas na vigilância à saúde para que mortes por doenças reconhecidamente evitáveis possam ser reduzidas.

Portanto, a assistência ao nascimento deve ocorrer de forma adequada buscando, garantir a integridade física e psíquica deste ser frágil e suplicante de cuidados, levando em consideração o processo de modificações na busca da homeostasia da vida extrauterina ${ }^{3}$.

Sendo assim, os indicadores da saúde da criança resultam da qualidade da assistência à saúde recebida pelas mães e crianças, e está associado tanto a fatores biológicos, quanto à assistência pré-natal, ao parto e ao recém-nascido. A redução dos índices de morbimortalidade infantil é um desafio não só para os serviços de saúde e a sociedade, já que a maioria das causas poderia ser evitada se houvesse melhores condições na atenção pré-natal, na assistência ao parto e ao neonato.

Dessa forma, deve-se realizar um conjunto de práticas voltadas para as condições/situações de risco durante o pré-natal, o parto e com o recém-nascido, estabelecendo estratégias que visem amenizar os riscos para o binômio mãe e filho. Para isso, são necessários o redirecionamento das políticas públicas e a melhoria da oferta de serviços públicos, que ofereçam uma adequada assistência durante o pré-natal, parto e ao neonato, evitando eventos indesejáveis e consequentemente buscando promoção de ações capazes de assegurar o bem-estar do recém-nascido após o parto. Este conjunto de ações contribui para a diminuição da taxa de mortalidade entre crianças que vão a óbito por causa da assistência obstétrica e neonatal oferecida, ou da sua ausência ${ }^{4}$.

sabido que altos índices de mortalidade infantil representam, de modo geral, neis precários de saúde e de desenvolvimento socioeconômico e se diminuídas dem amenizar más condições de vida em segmentos sociais específicos. Este icador contribui para avaliação da saúde e de desenvolvimento 
socioeconômico da população e para nos auxiliar processos de planejamento, gestão e avaliação de políticas e ações de saúde voltadas para a atenção pré-natal e ao parto, bem como para a proteção da saúde infantil 5 .

Portanto, as práticas de atenção à saúde voltada para a assistência maternoinfantil devem ser apoiadas na vigilância à saúde para que a morbimortalidade materno-infantil sejam reconhecidamente evitáveis. A partir disso, o presente estudo tem o objetivo de avaliar a qualidade da assistência do parto e a correlação com os indicadores de saúde da criança.

\section{Método}

Trata-se de um estudo quantitativo e avaliativo dos indicadores de morbidade e mortalidade materno e infantil realizado nas Unidades Básicas de Saúde que integram a rede da Atenção Primária à Saúde (APS), do município de Santa Cruz, Rio Grande do Norte, Brasil.

A população foi constituída por mulheres mães de crianças menores de dois anos (crianças com 1 ano, 11 meses e 29 dias) atendidas na rede APS. A escolha da amostra se deu por amostragem sistemática com base no cadastro das Unidades de Saúde da Família (USF), a partir do cálculo de uma prevalência de 19,3\% de mortalidade infantil em menores de dois anos no Estado do Rio Grande do Norte, adotando um erro permitido de $5 \%$ e grau de confiança de $95 \%$ e um poder de $80 \%$; resultando em $n=200$ mulheres, cadastradas na USF, mediante assinatura do Termo de Consentimento Livre e Esclarecido (TCLE) pela mãe ou responsável da criança.

Para coleta de dados foi utilizado um questionário elaborado conforme os pilares da APS entendida em elementos estruturados da assistência pré-natal, lidado pelo Ministério da Saúde e utilizado na Chamada Neonatal. As trevistas foram realizadas durante o período de julho a setembro de 2013. 


\section{ciência
pural}

Para análise dos dados foi utilizada a estatística descritiva por meio do conhecimento de médias, medianas, desvio padrão, frequências absolutas e relativas. Foi realizado ainda a análise bivariada e cálculo de testes estatísticos com a utilização do Statistical Package for the Social Sciences. Os dados foram organizados em tabelas e discutidos posteriormente à luz de literatura especifica. O projeto foi submetido ao comitê de Ética em Pesquisa da Faculdade de Ciências de Saúde do Trairí - UFRN e aprovado com número de parecer nº 311.613.

\section{Resultados}

No que se refere à idade das mães entrevistadas, obteve-se como média 28,17 anos; mediana 27,0 e desvio padrão de 7,22; a idade mínima foi 15 anos e máxima 45 anos. No tocante à escolaridade da mãe, a média em anos estudados foi de 6,04 anos; mediana 7,0 e desvio padrão de 3,28.

A média de idade da criança em meses foi de 10,42; a mediana de 10,0 e o desvio padrão de 6,49. Quanto à realização do pré-natal, 95,5\% (n=191) afirmaram ter realizado o acompanhamento, já 4,5\% (n=09) não realizaram. Em relação ao peso ao nascer verificou-se como média 3.292,62g, mediana de 3.297,50g e o desvio padrão de 652,18g. 
Tabela 1. Distribuição da classificação da qualidade do atendimento no parto. Santa Cruz/RN, Brasil, 2014.

\begin{tabular}{lccccccc}
\hline & Muito boa & Boa & Satisfatória & Ruim & $\begin{array}{c}\text { Muito } \\
\text { Ruim }\end{array}$ & Total \\
\hline $\begin{array}{l}\text { Qualidade } \\
\begin{array}{l}\text { atendimento } \\
\text { parto }\end{array}\end{array}$ no & $27,0 \%$ & $60,5 \%$ & $4,5 \%$ & $3,5 \%$ & $3,5 \%$ & $99,0 \%$ \\
$(\mathrm{n}=54)$ & $(\mathrm{n}=121)$ & $(\mathrm{n}=9)$ & $(\mathrm{n}=7)$ & $(\mathrm{n}=7)$ & $(\mathrm{n}=198)^{*}$ \\
\hline
\end{tabular}

Observou-se na tabela 1, a classificação da qualidade do atendimento no parto na ótica de usuários, onde 27,0\% $(n=54)$ afirmaram ter sido muito boa; $60,5 \%$ $(n=121)$ verbalizaram ter sido apenas boa; 4,5\% ( $n=9)$ satisfatória; 3,5\% (n=7) afirmaram ter sido ruim e 3,5\% $(n=7)$ consideraram muito ruim.

Tabela 2 - Distribuição dos indicadores de saúde da criança e correlação com a variável de classificação da qualidade do atendimento no parto. Santa Cruz/RN, Brasil, 2014.

\begin{tabular}{|c|c|c|c|}
\hline Variáveis & Sim & Não & Valor p \\
\hline $\begin{array}{l}\text { Criança colocada no peito após o } \\
\text { nascimento }\end{array}$ & $74,5 \%(n=149)$ & $23,5 \%(n=47)$ & 0,301 \\
\hline $\begin{array}{l}\text { Mãe e bebê permaneceram juntos } \\
\text { após o parto }\end{array}$ & $90,0 \%(n=180)$ & $9,0 \%(n=18)$ & 0,671 \\
\hline Bebê recebeu vacinas antes da alta & $93,0 \%(n=186)$ & $5,5 \%(n=11)$ & 0,108 \\
\hline Fez teste do pezinho & $95,0 \%(n=190)$ & $5,0 \%(\mathrm{n}=10)$ & 0,656 \\
\hline $\begin{array}{l}\text { A criança ficou internada no primeiro } \\
\text { mês de vida }\end{array}$ & $8,5 \%(n=17)$ & $91,5 \%(n=183)$ & 0,274 \\
\hline $\begin{array}{l}\text { Recebeu visita de algum profissional } \\
\text { de saúde }\end{array}$ & $65,0 \%(n=130)$ & $33,5 \%(n=67)$ & 0,022 \\
\hline
\end{tabular}

Na tabela 2, quando as mães foram questionadas em relação a criança ter sido colocada no peito após o nascimento, 74,5\% (n=149) afirmaram que sim e $23,5 \%(n=47)$ afirmaram não ter sido colocada. No que se refere à mãe e bebê terem ficados juntos no quarto após o parto, 90\% (n=180) confirmaram ter ficado juntos no quarto após o parto e $9 \%(n=18)$ não ficaram juntos.

Em relação ao recebimento das vacinas antes da alta hospitalar 93\% (n=186) afirmaram ter recebido as vacinas e 5,5\% (n=11) afirmaram não ter recebido. Quanto ao teste do pezinho 95\% $(n=190)$ alegaram ter feito e 5\% $(n=10)$ alegaram não ter sido realizado. 
Em relação da criança ficar internada no primeiro mês de vida 8,5\% (n=17) afirmaram que precisaram de internação e 91,5\% (n=183) afirmaram não ter precisado.

No se refere à visita de algum profissional de saúde no domicilio na primeira semana após o parto 65\% $(n=130)$ alegaram ter recebido a visita e 33,5(n=67) alegaram não ter recebido a visita.

No que se refere a qual profissional de saúde realizou a visita domiciliar na primeira semana após o parto, 42,5\% $(n=85)$ afirmaram ter recebido visita do agente comunitário de saúde, 0,5\% $(n=1)$ afirmaram ter recebido a visita do médico, 25,5\% ( $n=51)$ afirmaram ter recebido a visita do enfermeiro, 13\% ( $n=26)$ alegaram não ter recebido a visita, 16,5\% $(n=33)$ alegaram ter recebido visita do agente comunitário de saúde e enfermeiro juntos, $0,5 \%(n=1)$ alegaram ter recebido a visita do agente comunitário de saúde, médico e enfermeiro juntos, 0,5\% ( $n=1)$ afirmaram ter recebido visita do médico e enfermeiro juntos e 1\% (n=2) afirmaram ter recebido a visita do médico e agente comunitário de saúde juntos.

\section{Discussão}

Este estudo da qualidade da assistência do parto e avaliação do impacto nos indicadores da saúde da criança, mostra que o atendimento prestado às mães no parto e ao recém-nascido foi realizado de forma satisfatória, seguindo o que é preconizado pelo Ministério da Saúde, abrangendo um atendimento de forma igualitária, integralizada e humanizada. Sendo assim, fica perceptível a importância do atendimento adequado tanto na hora do parto como os primeiros cuidados com os recém-nascidos, pois uma assistência de boa qualidade para o binômio mãe-filho contribui significativamente para a redução da norbimortalidade materna e infantil.

Conforme mostra a tabela 1, a qualidade da assistência ao parto ocorre no ral de forma satisfatória, atendendo de forma adequada e humanizada as 
necessidades das parturientes de acordo com o que é preconizado pelo Ministério da Saúde, evitando assim situações indesejadas que causem risco para a mãe e o bebê.

Desse modo, é necessário que ocorra a humanização do parto onde ocasiona mudanças de modos e de rotinas, utilizando-se práticas assistenciais que garantam o bem estar do binômio mãe-filho. A consequência de uma assistência não humanizada e não resolutiva impacta de forma negativa nos índices de morbimortalidade materna e perinatal. Contudo, nos últimos tempos têm surgido programas na saúde em prol de uma assistência mais digna, humanizada e holística em que se considera a pessoa como um todo e não como um objeto.

Para tanto, é necessário que ocorra a conquista de profissionais com qualidade e que sejam comprometidos de forma pessoal e profissional, que acolham a mulher com respeito, ética e dignidade. A cautela profissional à gestante e/ou parturiente representa um item fundamental para adquirir bons resultados, tanto maternos quanto perinatais. Dessa forma, as melhores qualidades da aplicação profissional e institucional ao parto têm a capacidade de amenizar a ocorrência de morbimortalidade materna e infantil ${ }^{6}$.

Nesse sentido, o parto é um dos momentos decisivos para a sobrevivência materna e infantil. Quando os índices se encontram elevados, faz com que surja questionamento inevitável de que as políticas de gênero não têm conseguido resultado tão significativo. Isso indica que o acesso de forma adequada e organizada aos serviços de saúde e uma boa qualidade no atendimento são fundamentais na prevenção dessas mortalidades. Entretanto, a persistência de elevados índices de mortalidade materna e neonatal ao longo dos anos sinaliza para a necessidade de análise das medidas de implementação de políticas públicas para a melhoria da qualidade da atenção à saúde da mulher e da criança7.

Desta forma, uma assistência adequada à gestação e ao parto é essencial a reduzir os índices de morbimortalidade materno-infantil. É necessário 
qualificar o cuidado, além de aumentar o acesso aos serviços de saúde, desde o pré-natal até assistência ao parto e ao nascimento, conservando a continuação da atenção ao recém-nascido e à puérpera identificando os riscos e evitando problemas de morbidade e mortalidade do binômio mãe-filho.

$\mathrm{Na}$ tabela 2, onde fala dos cuidados com a criança após o nascimento, podese perceber que, a maioria afirmou que a criança foi colocada no peito após o parto. No que se refere à mãe e bebê terem ficados juntos no quarto após o parto, a maioria alegou ter ficado juntos no quarto.

Quanto ao aleitamento materno na primeira hora de vida do bebê, sabe-se que é fundamental tanto para o bebê como para a mãe, pois promove o contato pele a pele, e com isso aumenta o vínculo afetivo da mãe e filho, além disso, o leite materno nos primeiros dias pós-parto, chamado de colostro, é o leite ideal nos primeiros dias de vida e estimula o crescimento e desenvolvimento da criança de forma adequada.

A maioria das usuárias afirmou ter colocado o bebê no peito na primeira hora de vida, houve ainda uma quantidade significativa das entrevistadas que não colocaram no peito, havendo assim uma necessidade de os profissionais de saúde aumentarem o estímulo ao aleitamento materno em seguida ao nascimento.

Isso mostra que os profissionais de saúde devem incentivar cada vez mais o fortalecimento das ações de proteção, promoção, e apoio ao aleitamento materno, devendo ser iniciado durante o pré-natal, em seguida após o parto, puerpério e nas consultas de crescimento e desenvolvimento da criança. $\mathrm{O}$ leite materno deve ser exclusivo até o sexto mês, e, a partir do sexto mês até dois anos de vida, a alimentação deve ser complementada com outros alimentos ${ }^{8}$.

Em relação à permanência do bebê com a mãe após o parto, observou-se e em apenas uma minoria (9\%) - mãe e filho não permaneceram juntos após o arto. Embora seja uma quantidade mínima, tem um valor expressivo para que os fissionais de saúde fiquem atentos para fornecer uma melhora na assistência a 
esse binômio, pois, essa permanência conhecida como, alojamento conjunto é uma regra hospitalar em que o neonato sadio, após o nascimento, fica com a mãe, 24h por dia, em um mesmo espaço, até a alta hospitalar. Este princípio permite a prestação de todos os cuidados assistenciais, além de ser um direito de a mãe permanecer junto ao seu filho.

Para manter esse vínculo com a criança, é prevista a prática do Alojamento Conjunto (AC), que é um sistema hospitalar em que o recém-nascido sadio permanece com a mãe logo após o nascimento, 24 horas por dia, até a alta hospitalar. Dessa forma, os profissionais têm como fornecer uma assistência de boa qualidade tanto para a mãe como para o filho e atender as necessidades de ambos até o momento da alta. É de suma importância estabelecer um vínculo entre o profissional e a puérpera, pois, se esta estiver satisfeita com a assistência, os objetivos do alojamento conjunto poderão ser alcançados, em benefício da mãe e do bebê, como fortalecer o vínculo entre mãe e filho, proteção e promoção ao aleitamento materno, entre outros ${ }^{9}$.

Quanto ao fato de a criança ter recebido vacinas antes da alta hospitalar, observou-se que a maioria afirmou ter recebido. Isso mostra uma quantidade expressiva, contudo, o sistema de saúde pública ainda necessita de uma supervisão direta, já que é proposta do Ministério da Saúde que as crianças antes da alta recebam as duas vacinas previstas no calendário vacinal básico, BCG e Hepatite B.

A vacina BCG é indicada para a prevenção da tuberculose em crianças menores de cinco anos de idade, com uma maior frequência em menores de um ano, o mais próximo possível do nascimento em crianças com o peso acima de 2.000 gramas. Sendo apenas contra indicada nos indivíduos HIV- positivos sintomáticos. O BCG tem um papel fundamental para o controle, especialmente locais com altos índices de tuberculose ${ }^{10}$. 
Já a imunização contra a hepatite B deve-se iniciar de preferência após o nascimento, nas primeiras 12 horas de vida, a fim de evitar a transmissão vertical. Caso isso não ocorra, deve-se iniciar o mais rápido possível, na unidade neonatal ou na visita ao Posto de Saúde. A vacina contra hepatite B poderá ser aplicada simultaneamente com outras vacinas do calendário vacinal de rotina, não apresentando comprometimento da eficácia ou aumento de eventos adversos ${ }^{11}$.

No tocante à realização do teste do pezinho, a maioria das mães relatou ter realizado o teste. Porém, ainda há necessidade de uma maior atenção qualificada desse teste, pois possui uma finalidade fundamental de identificação de bebês que possam desenvolver morbidades que originam a deficiência se não forem detectadas precocemente.

Dessa forma, distúrbios metabólicos e genéticos como o hipotireoidismo congênito e a fenilcetonúria são morbidades consideradas problemas de saúde pública por ocasionarem, na criança, deficiência mental se o diagnóstico for tardio. Daí passou a existir o Screening Neonatal, ou, como é chamado, "Teste do Pezinho", um exame laboratorial que é efetivado em amostras de sangue colhidas em papel filtro especial, de certa quantidade de gotas de sangue conseguidas de uma leve punção do calcanhar da criança, com o intuito de detectar precocemente erros do metabolismo e infecções congênitas que podem ser tratadas, amenizando sua repercussão sobre a saúde do recém-nascido. Deve ser efetivado a partir do quinto dia de vida. Com isso as equipes de saúde precisam ficar atentas às crianças que não aparecerem na unidade de saúde na primeira semana de vida e realizar neste caso uma busca ativa dessas crianças ${ }^{12}$.

No que se refere à internação da criança no primeiro mês de vida, apenas 3,5\% das mães alegaram ter precisado ficar internadas. Dessa forma, há uma ecessidade de a equipe de saúde ofertar uma atenção mais qualificada para evitar se tipo de situação e melhor promover a saúde da criança. 
As internações representam um indicador hospitalar de importante rastreamento da Atenção Primária à Saúde, pois mensura a taxa de hospitalização na primeira semana integral da criança ou a partir dela. Isso impulsiona uma atenção contínua para fins de prevenção da Taxa de Mortalidade Neonatal Precoce e Tardia. Com isso, é fundamental que os profissionais de saúde forneçam uma assistência mais qualificada e humanizada na hora do parto e os cuidados com o neonato para evitar situações indesejadas com a saúde da criança. É fundamental também que os profissionais de saúde, principalmente a equipe de enfermagem, proporcionem acompanhamento absoluto às famílias, com apoio e orientação sobre a situação de saúde do RN, deixando os familiares mais tranquilos, pois eles são importantes no processo de cura e de desenvolvimento e crescimento do bebêt13.

No que se refere ao recebimento da visita por parte de algum profissional no domicílio no período puerperal, houve uma quantidade expressiva de não recebimento de visita domiciliária. Desse modo, há uma necessidade de que os profissionais sejam mais responsáveis e sensibilizados para que forneçam uma atenção mais qualificada e humanizada, realizando a visita domiciliar e atendendo às necessidades da puérpera e do neonato, esclarecendo suas dúvidas, incentivando o aleitamento materno, orientando quanto aos cuidados com o recém-nascido, entre outros cuidados.

Desse modo, a importância das visitas domiciliárias, é um modo de cuidado mais humanizado à puérpera, pois possibilita um cuidado mais próximo e individualizado, respondendo suas dúvidas e assim potencializando seu desempenho como mãe. É imprescindível deixá-la expressar o que está vivenciando, para obter o maior número de informações que sejam fundamentais para o planejamento e execução do cuidado. A visita domiciliária, nesta situação, proporciona um modo especial de encontro entre o profissional e a puérpera junto família, especialmente por se tratar de um ambiente conhecido ${ }^{14}$. 
A partir disso, pode-se perceber que é fundamental fornecer uma assistência de qualidade ao parto, para prevenir morbidades e mortalidade materna e consequentemente ocasionar um impacto positivo nos indicadores na saúde da criança. Para tanto, faz-se necessária a qualificação, conscientização e humanização dos profissionais de saúde, para fornecer uma assistência de qualidade e assim diminuir as taxas de mortalidade materna e infantil e respectivamente aumentar a expectativa de vida da população.

\section{Conclusões}

Pode-se perceber neste estudo que a assistência ao parto acontece de forma adequada e satisfatória, atendendo necessidades da maioria das entrevistadas, e consequentemente gerando um impacto positivo nos indicadores da saúde da criança discutidos neste presente estudo. Porém ainda há uma necessidade de conscientização e qualificação dos profissionais de saúde, para que não ocorram situações indesejáveis na saúde materno-infantil e diminua as taxas de morbidade e mortalidade materna e neonatal; principalmente no que se diz respeito às visitas domiciliares, pois a maioria das entrevistadas relatou não ter recebido visita domiciliar, o que mostra a não continuidade da assistência materno-infantil.

Portanto há uma necessidade de melhorar a qualidade, continuidade, e fornecer uma assistência cada vez mais digna e adequada, já que é direito da população ter uma assistência de saúde qualificada e dever dos profissionais de saúde de fornecê-la adequadamente.

Essa iniciativa se encontra em consonância com o que está posto pelo Ministério da Saúde e pela Organização Mundial da Saúde quando fornece maiores condições de atenção à saúde, entendida em promoção de cursos de especialização/capacitação para correção das fragilidades da assistência ospitalar nas maternidades do Brasil, bem como vem investindo em melhoria na fraestrutura e equipamentos para garantir um nascimento seguro e com 
qualidade, com menores riscos de mortalidade e com vistas de identificação de prováveis complicações para a saúde da mãe e criança.

\section{Referências}

1. Nascimento RM, Leite AJM, Almeida NMGS, Almeida PC, Silva CF. Determinantes da mortalidade neonatal: estudo caso-controle em Fortaleza, Ceará, Brasil. Cadernos de Saúde Pública, 2012; 28(3): 559-72.

2. Brasil. Ministério da Saúde. Secretaria de Vigilância em Saúde. Secretaria de Atenção à Saúde. Manual de Vigilância do Óbito Infantil e Fetal e do Comitê de Prevenção do Óbito Infantil e Fetal. 2a ed. Brasília: DF. Ministério da Saúde; 2009.

3. Souza TG, Gaiva MAM, Modes PSSA. A humanização do nascimento: percepção dos profissionais de saúde que atuam na atenção ao parto. Rev. Gaúcha Enferm. 2011; 32(3).

4. Oliveira RB, Melo ECP, Knupp VMAO. Perfil dos óbitos Infantis no Município do Rio de Janeiro Segundo Peso ao Nascer, no Ano de 2002. Esc Anna Nery. Rev. Enferm. 2008; 12(1):25-9.

5. Stábile AP, Braz JC, Furtado MCC, Mello DF. Indicadores de saúde infantil na estratégia saúde da família no Brasil: revisão integrativa da literatura Rev. Cinc. Md. 2013;22(1):31-41.

6. Ferrari RAP, Bertolozzi MR. Mortalidade pós-neonatal no território brasileiro: uma revisão da literatura. Rev. esc. enferm. USP. 2012;46(5).

7. Cecatti JG, Calderon IMP. Intervenções benéficas durante o parto para a prevenção da mortalidade materna. Rev. Bras. Ginecol. Obstet. 2005; 27(6).

8. Rocci Eliana, Fernandes RAQ. Dificuldades no aleitamento materno e influência no desmame precoce. Rev. bras. enferm. 2014;67(1):22-27. 


\section{ciênncia
pural}

9. Odinino NG, Guirardello EB. Satisfação da puérpera com os cuidados de enfermagem recebidos em um alojamento conjunto. Texto contexto - enferm. 2010;19(4):682-690.

10. Barreto ML, Pereira SM, Ferreira AA. Vacina BCG: eficácia e indicações da vacinação e da revacinação. J. Pediatr. 2006;82(3):45-54.

11. Divisão de Imunização. Vacina contra hepatite B. Rev. Saúde Pública. 2006; 40(6): 1137-1140.

12. Reichert APS. Conhecimento de mães quanto a importância do teste do pezinho. Rev. bras. enferm. 2003; 56(3): 226-229.

13. Centa ML, Moreira EC, Pinto MNGHR. A experiência vivida pelas famílias de crianças hospitalizadas em uma unidade de terapia intensiva neonatal. Texto contexto - enferm. 2004; 13(3): 444-451.

14. Bernardi MC, Carraro TE, Sebold LF. Visita domiciliária puerperal como estratégia de cuidado de enfermagem na atenção básica: revisão integrativa. Rev. Rene. 2011;12.1074-80. 